\title{
The search of the anisotropy of the primary cosmic radiation by the difference method
}

\author{
Victor Pavlyuchenko ${ }^{1, \text { a }}$, Romen Martirosov ${ }^{2}$, Natalia Nikolskaya ${ }^{1}$, and Anatoly Erlykin ${ }^{1}$ \\ 1 P.N.Lebedev Physical Institute, Moscow 119991, Russia \\ 2 A Alikhanian National Laboratory, Yerevan, Armenia
}

\begin{abstract}
On the basis of experimental data obtained in the knee energy region with the GAMMA array an anomaly has been found in the mass composition of primary cosmic rays coming from the region of the VELA cluster. We used an original difference method which has high sensitivity, stability against accidental experimental errors and the possibility to separate anomalies connected with the laboratory coordinate system from anomalies observed in the celestial coordinates. The multiple scattering of the charged particles in the galactic magnetic fields makes it possible to study regions of the sky outside the direct visibility of the array.
\end{abstract}

\section{Introduction}

The origin of the knee at energies of $3-4 \mathrm{PeV}$ in the all-particle spectrum of the primary cosmic rays (PCR) has caused interest since its discovery in 1958 [1] because it is important for understanding the mechanism of PCR acceleration in the Galaxy. At the present time there are three main astrophysical models which try to explain the origin of the knee in this energy region. The selection between them can be made studying the anisotropy of arriving PCR particles. These models are the classic diffusion model [2], the model of the limited maximum energy for particle acceleration in the shells of supernova remnants [3] and the model of a nearby source, particles from which overlap with the smooth galactic spectrum, create an excess in the $\mathrm{PeV}$ region and imitate the formation of the knee [4]. This source has to be sufficiently close to us in order for its particles to have insufficient time to mix with particles from other sources.

So far there is no reliable experimental confirmation of the validity of these models, since the measured amplitude of the anisotropy in the $0.1-1 \mathrm{PeV}$ region does not exceed $0.1 \%$ [5]. This is mainly caused by the mixing of the particle trajectories in chaotic magnetic fields due to which the propagation of PCRs in the Galaxy is similar to the large-scale diffusion transport. However, it is possible to study not only an anisotropy of the PCR intensity, but also the anisotropy of the mass composition since for the same conditions nuclei with different masses scatter differently in the same magnetic fields. In this case the multiple scattering of particles does not prevent, but rather helps to study PCRs from sources outside the direct visibility of the array. To do so we searched for regions of the sky having the maximum difference of the mass composition for particles coming from the opposite directions.

a e-mail: pavict@rambler.ru

\section{The difference method}

The main idea of the method [6] is the following: we take directions $\left(\ell_{0}, b_{0}\right)$ in the galactic coordinate system and build a cone around each of them. Its width angle $\theta$ (or $\left.H_{0}=\cos \theta\right)$ is selected in such a way that it divides all registered particles in two sets with an equal number of particles in both of them - inside and outside the cone: $n^{\text {in }}=n^{\text {out }}$. In both sets we build the distributions of the parameter depending on the mass composition and then both distributions are subtracted from each other. The difference between both distributions is evaluated by the standard estimate of $\chi^{2} / J=J^{-1} \Sigma_{i}\left(\Delta_{i} / \sigma_{i}\right)^{2}$. Here $J$ is the number of degrees of freedom, $\Delta_{i}=m_{i}^{\text {in }}-m_{i}^{\text {out }}$ is the difference between distributions in the interval ' $i$ ' and its error $\sigma_{i}=\sqrt{m_{i}^{\text {in }}+m_{i}^{\text {out }}+1}=\sqrt{n_{i}+1}$ is calculated from the Poisson distribution. If $n^{\text {in }}=n^{\text {out }}$ then there is no need to use the normalisation for the subtraction and the total number of events $n_{i}$ in the interval ' $i$ ' does not depend on the galactic coordinates $\left(\ell_{0}, b_{0}\right)$. It is crucially important for the comparison of $\chi^{2} / J$ values with each other when searching for their maximum. One can see from the above formulae that $\chi^{2} / J$ values calculated for the $\left(\ell_{0}, b_{0}\right)$ and the opposite $\left(\ell_{0}+180^{\circ},-b_{0}\right)$ directions are equal. That is why the equation $n^{\text {in }}=n^{\text {out }}$ allows us to study the entire sky within the sensitivity of the method using the array with the limited fiducial sector. However, it does not allow to distinguish the $\left(\ell_{0}, b_{0}\right)$ direction from the opposite one, $\left(\ell_{0}+180^{\circ},-b_{0}\right)$.

In order to divide the total number of events in two sets for the studied $\left(\ell_{0}, b_{0}\right)$ direction we used the expression $H=\cos \psi=\sin b_{0} \cdot \sin b+\cos b_{0}$. $\cos b \cdot \cos \left(\ell-\ell_{0}\right)$. Here $H$ is the cosine of the angle $\psi$ between $(\ell, b)$ - the arrival direction of the particle and $\left(\ell_{0}, b_{0}\right)$ - the studied direction in the spherical coordinate system. Events with $H>H_{0}$ enter the interiors of the cone and are included in $n^{i n}$. Those with $H<H_{0}$ enter the outer regions of the cone and are included in $n^{\text {out }}$. The common background and possible methodical errors are equal in 


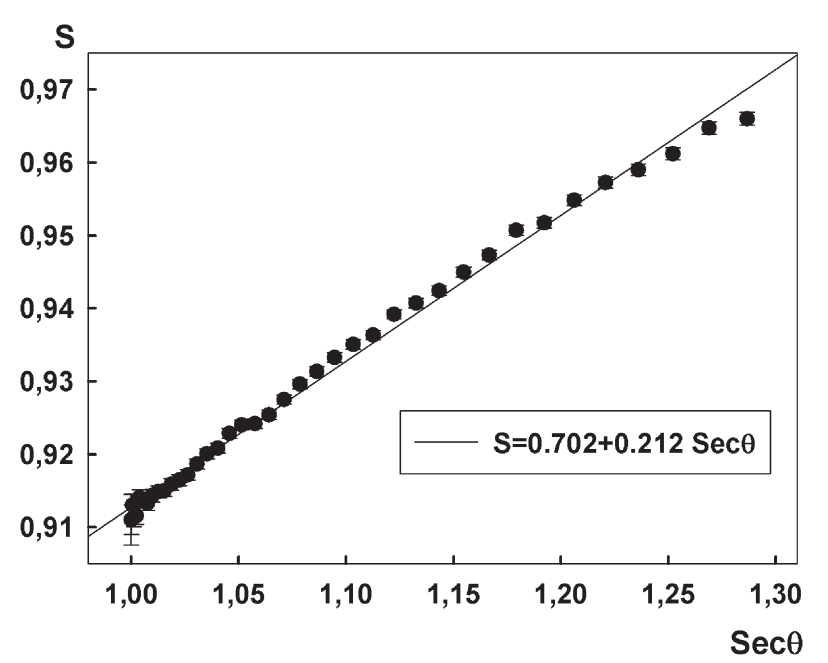

Figure 1. The experimental dependence of the lateral age parameter $S$ from $\sec \theta, \theta$ being the zenith angle of the EAS arrival direction. The straight line is a linear fit of this dependence by the expression shown in the inset.

both sets of events and if $n^{\text {in }}=n^{\text {out }}$ they disappear in the process of the subtraction.

The requirements for the experimental array are common: it has to be stable and its registration efficiency should not depend on the azimuth of the particle arrival direction.

\section{Results}

The blind search of the anisotropy in the PCR mass composition has been made with experimental data $\left(3.38 \cdot 10^{6}\right.$ events) obtained with the extensive air shower (EAS) array GAMMA, operated at an altitude of $3200 \mathrm{~m}$ above sea level on Mount Aragats in Armenia. Its geographical coordinates are $40.28^{\circ} \mathrm{N}$ and $44.11^{\circ} \mathrm{E}$. The array consists of a system of surface and underground detectors. The detailed description of the array, its technical characteristics and main results are given in [7-10].

The PCR mass composition at high energies is determined with large uncertainties by indirect methods studying various EAS characteristics and model dependent simulations. In this study we used the lateral age, $S$, of EAS where $\mathrm{S}$ is the experimental dimensionless parameter connected with the steepness of the lateral distribution function (LDF) of the charged EAS particles approximated by the so called NKG function [11].

The age $S$ depends on the mass composition since PCR nuclei heavier than protons interact higher in the atmosphere for the same conditions. The cascade development of EAS initiated by these nuclei is faster and at the observation level EAS have wider LDF with bigger $S$. The $S$ parameter is one of the basic shower parameters, which is well determined in experiments without an involvement of models. In Fig. 1 we show the measured experimental dependence of the $S$ parameter on the zenith angle $\theta$. With increasing $\theta$ the geometrical path length from the first interaction point to the array also increases and also the thickness of the atmosphere above the array as $X=X_{0} \sec \theta\left(X_{0}=700 \mathrm{~g} \mathrm{~cm}^{-2}\right.$ is the vertical thickness). The near linear growth of $S$ with $\sec \theta$ in the zenith range

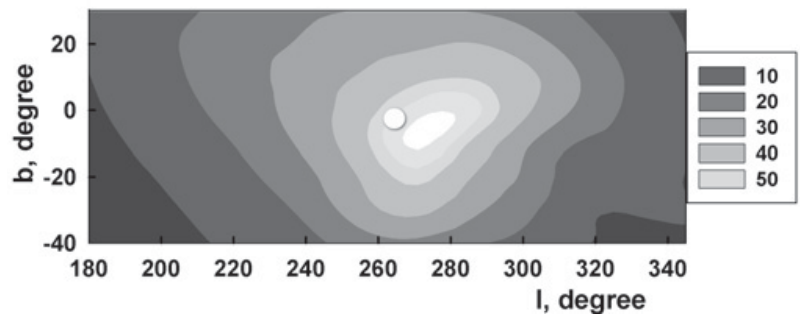

Figure 2. The dependence of $\chi^{2} / J$ value which evaluates the difference between the $S$-distributions in two sets of EAS coming from the opposite directions on galactic coordinates. The white circle in the center -the position of the Vela cluster.

from $0^{\circ}$ to $40^{\circ}$ justifies the good sensitivity of $S$ to $\theta$ and therefore to the PCR mass composition.

We have made a blind search for anomalies in the vicinity of the galactic plane $(b=0)$ in the range of galactic latitudes $-40^{\circ} \leq b \leq 40^{\circ}$ and in the full range of galactic longitudes from $\ell=0^{\circ}$ to $360^{\circ}$. A single maximum of $\chi^{2} / J=57.3$ for $J=17$ has been found in the direction of $\ell_{m}=277.3^{\circ} ; b_{m}=-5.3^{\circ}$ (Fig. 2). Using the normalized fit of the two-dimensional $\ell, b$ distribution then the coordinates of the maximum are $\ell_{m}=270 \pm$ $09^{\circ} ; b_{m}=-0.1 \pm 0.8^{\circ}$

The value of $\chi^{2} / J=57.6$ with $J=17$ is very high. For random fluctuations $\chi^{2} / J$ has to be close to $1 \pm \sqrt{2 / J}$. By dividing the total number of events by $2,3,5$ and 10 we ensured that the big value of $\chi^{2} / J$ is due to the large statistics and the high sensitivity of the method, which used the full statistics in each point of the sky. When dividing the statistics the maximum value of $\chi^{2} / J$ decreases from 57.6 to $29.4,20.3,12.6$ and 7.9 respectively, i.e. with a nearly invariable shape of the distributions the value of $\chi^{2} / J-1$ depends linearly on the total number of events in our case.

An additional study [12] showed that showers from the maximum region are initiated by PCR with a lighter mass composition ('young' EAS) compared with those from the opposite direction at $\mathrm{PeV}$ energies. Since light nuclei at a given energy are less deviated in magnetic fields than heavier ones it means that the source of the anomaly is in the direction of the maximum. The analysis of the stability of this result [12] showed that accidental errors of the $S$ parameter do not change the position of the anomaly but reduce the hight of the maximum $\chi^{2} / J$, which depends on the error values. If the errors are asymmetric in azimuth angles of the laboratory coordinate system, which rotates with the Earth, then they induce the appearence of an additional maximum in the direction of the Earth's rotation axis ('World's Pole').

Thus the difference method not only supresses background and methodical uncertainties but allows to separate effects connected with the laboratory coordinate system from those connected with the system of fixed celestial coordinates. The asymmetry in azimuth angles is revealed by the appearence of the peak at the Pole and a stable anomaly in other directions is most likely related to celestial coordinates. In the difference method there are no model assumptions.

Nearby the found anomaly there is the Vela cluster with closely located supernova remnants (SNR): the central Vela SNR and two others - Vela X $\left(263.9^{\circ},-3.3^{\circ}\right)$ and 
Vela $\operatorname{Jr}\left(266.2^{\circ},-1.2^{\circ}\right)$, the distances from which are about 0.2 and $0.3 \mathrm{k} \mathrm{pc}$ respectively. It is close to the anomaly's coordinates (see Fig. 2) and located at a relatively close distance to the Earth. Evidently this cluster is a good candidate for being a nearby PCR source. However, the difference method does not allow to calculate their PCR intensity and to tell whether it is completely responsible for the formation of the knee.

\section{Conclusion}

The difference method demonstrated its simplicity, high sensitivity and stability against accidental errors. From the very beginning it accounts for the PCR diffusive transport and the rotation of the Earth. That is why it is able to survey the whole celestial sphere using the experimental array with a limited fiducial sector and to separate anomalies connected with the laboratory coordinate system from anomalies in celestial coordinates.

Using this method we have found an anomaly of the PCR mass composition in the knee energy region with a high statistical security. Closest to this anomaly is the Vela cluster of SNR, which can be regarded as a good candidate for being the nearby single source responsible for the formation of the knee.

This work is done with the financial support from the Program of Fundamental Research of the Presidium of the Russian Academy of Sciences 'High energy physics and neutrino astrophysics'.
Authors are very thankful to Dr.B.Pattison for his help in the preparation of the paper and useful discussions.

\section{References}

[1] G.V. Kulikov, G.B. Khristiansen, Journ. Exp. Theor. Phys. 35, 635 (1958) (in Russian)

[2] V.S. Ptuskin, Usp. Fiz. Nauk 177, 558 (2007) (in Russian)

[3] E.G. Berezhko, H. Völk, Astron. Astrophys. 451, 981 (2006)

[4] A.D. Erlykin, A.W. Wolfendale, J. Phys. G: Nucl. Part. Phys. 23, 979 (1997)

[5] G. Guillian et al., Phys. Rev. D 75, 062003 (2007)

[6] V.P. Pavlyuchenko, Bull. Lebedev Phys. Inst. 41/3, 55 (2014)

[7] A.P. Garyaka et al., Astropart. Phys. 28, 169 (2007)

[8] A.P. Garyaka et al., J. Phys. G:Nucl. Part. Phys. 35, $115201(2008)$

[9] R.M. Martirosov et al., Proc. 32 Int. Cosm. Ray Conf., Beijing 1, 178 (2011)

[10] A.P. Garyaka et al., J. Contemp. Phys. Armenian Acad. Sci. 48/2, 51 (2014)

[11] C. Hayakawa, Cosm. Ray Phys: Nucl. Astrophys. Aspect, Wiley (1969)

[12] V.P. Pavlyuchenko et al., Bull. Lebedev Phys. Inst. 5, 55 (2014) arxiv1406.0799; 1509.00316 